\title{
Dispositivos de la sensibilidad: Para una tecnología de la experiencia estética
}

\section{Artículo de investigación}

\section{Inés Pérez-Wilke}

Grupo de Investigación Transdisciplinar Semeruco, Caracas, Venezuela inespewi@yandex.com

Recibido: 26 de abril de 2020

Aprobado: 10 de septiembre de 2020

Cómo citar este artículo: Pérez-Wilke, Inés (2021). Dispositivos de la sensibilidad: Para una tecnología de la experiencia estética. Calle 14: revista de investigación en el campo del arte 16(29). pp. 146-161.

https://doi.org/10.14483/21450706.17409

Este artículo recoge una línea reflexiva producida en el transcurso de los últimos dos años de trabajo en el Grupo de Investigación Transdisciplinar Semeruco, el cual reúne estudiantes, docentes y colaboradores en torno a la práctica de la improvisación escénica interdisciplinar, lo cual ha implicado una amplia reflexión en torno a las artes performativas. Agradezco al todo el equipo que me ha acompañado en este transcurso de complicidades, riesgos y hallazgos.

\section{(2) (1)}

https://creativecommons.org/licenses/by/4.0/deed.es 


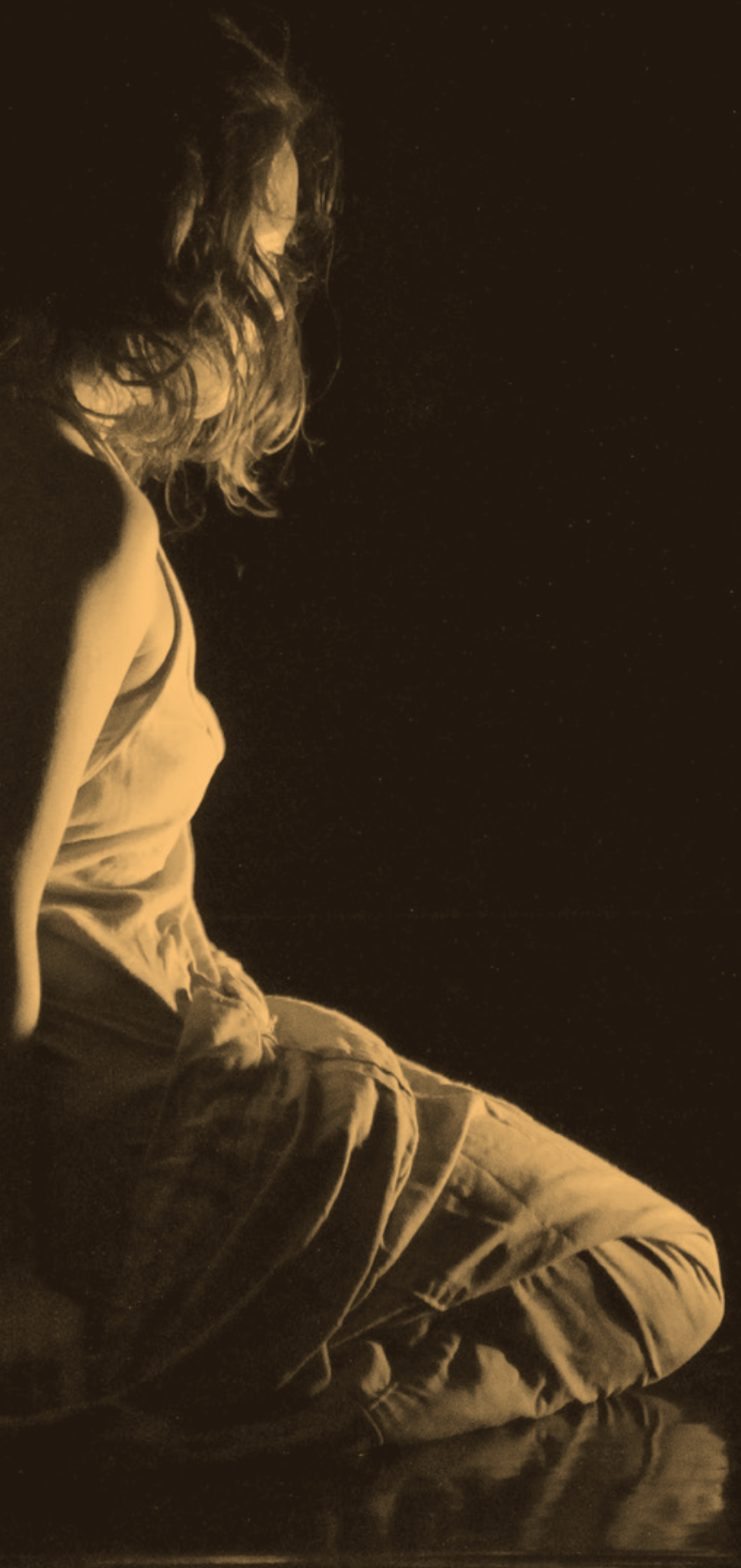




\title{
Resumen
}

La comprensión de la experiencia estética como instancia nuclear en la producción de subjetividades y la construcción de realidad social abre una reflexión sobre la dimensión tecnológica del trabajo sensible. Esta sería una forma de producción y trasformación de lo real, que aquí opera como trasvase creativo entre lo material y lo simbólico entendido como tecnologías de lo sensible. Para ello se parte del lugar de la mediación estética en las dinámicas vitales de nuestros pueblos, se muestran algunos dispositivos estéticos tradicionales, y su vinculación con artefactos del arte contemporáneo para la operación estética y la creación común de lo real. En el caso de las prácticas creadoras, artísticas y/o estéticas este proceso se orienta a la producción material de una posibilidad existencial, que pueda ser habitada, alcanzando una dimensión política.

\section{Palabras Clave}

Experiencia estética; arte popular; estéticas descoloniales; tecnologías estéticas; ritual

\section{Sensitivity Devices. For a Technology of Aesthetic Experience}

\begin{abstract}
The understanding of the aesthetic experience as a core instance in the production of subjectivities and the construction of social reality opens a reflection on the technological dimension of work with the senses. This would be a form of production and transformation of the real, which here operates as a creative transfer between the material and the symbolic, seen as a technology of the sensible. To do this, we start from the place of aesthetic mediation in the vital dynamics of our peoples, and show some traditional aesthetic devices and their link with contemporary artifacts for the aesthetic operation and the common creation of the real. In the case of creative, artistic and/or aesthetic practices, this process is oriented towards the material production of an existential possibility, which can be inhabited, reaching a political dimension.
\end{abstract}

\section{Keywords}

Aesthetic experience; popular art; decolonial aesthetics; aesthetic technologies; ritual

Dispositifs de la sensibilité. Pour une technologie de l'expérience esthétique

\section{Résumé}

La compréhension de l'expérience esthétique comme instance centrale dans la production de subjectivités et la construction de la réalité sociale ouvre une réflexion sur la dimension technologique du travail avec les sens. Ce serait une forme de production et de transformation du réel, qui opère ici comme un transfert créatif entre le matériel et le symbolique, vu comme une technologie du sensible. Pour ce faire, nous partons de la place de la médiation esthétique dans la dynamique vitale de nos peuples, et montrons quelques dispositifs esthétiques traditionnels et leur lien avec des artefacts contemporains pour l'opération esthétique et la création commune du réel. Dans le cas des pratiques créatives, artistiques et / ou esthétiques, ce processus s'oriente vers la production matérielle d'une possibilité existentielle, qui peut être habitée, atteignant une dimension politique.

\section{Mots clés}

Expérience esthétique ; art populaire ; esthétique décoloniale ; technologies esthétiques ; rituel 


\section{Resumo}

A compreensão da experiência estética como instancia nuclear na produção de subjetividades e a construção de realidade social abre uma reflexão sobre a dimensão tecnológica do trabalho sensível. Esta seria uma forma de produção e transformação do real, que aqui opera como transferência criativa entre o material e o simbólico entendido como tecnologias do sensível. Por isso se parte do lugar da mediação estética nas dinâmicas vitais de nossos povos, se mostram alguns dispositivos estéticos tradicionais e, sua vinculação com artefatos da arte contemporânea para a operação estética e a criação comum do real. No caso da práticas criadoras, artísticas e/ ou estéticas este processo se orienta à produção material de uma possibilidade existencial, que possa ser habitada, alcançando um dimensão política.

\section{Palavras chave}

Experiência estética; arte popular; estéticas decoloniais; tecnologia estéticas; ritual

\section{Imapas tarisunchi kunauramandata iachaikungapa ruraskata}

\section{Maillallachiska}

Iuiachiku tukuikunata imami ñugpata iachaskakuna iachachinga kunaura, kawanchi imapas tukuikuna llapa llakichinakuspa kausanchi. Kai runa churaku paipa makiwa ruraskata mana rungaringapai nispa chasalla sakingapa. Panaapi churaska kami pai kikin kausaskata chimanda ni kansi llapa unaimandata iuiarispa pangapi kikaska.

\section{Rimangapa ministidukuna}

Ruraita katichidur; ruraita apachii; ruraikuna ñugpata; kunauramanda ruraikuna; kaugsaita paini 


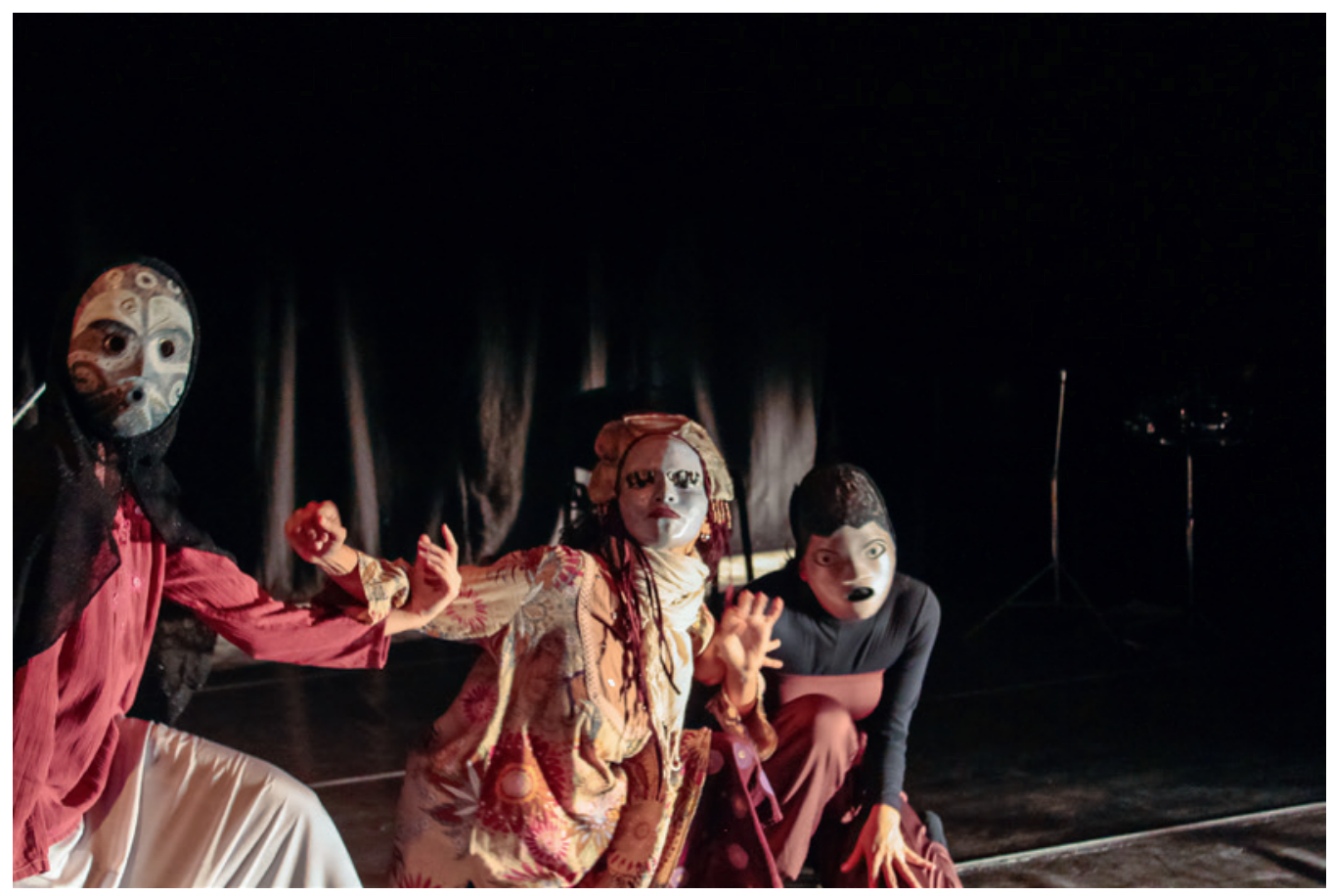

Imagen 1. Semeruco.Git en Tríadas Improvisación al borde. Caracas -Venezuela. 2020. Foto María Angélica Bermúdez. Archivo del grupo

\section{Introducción}

Necesitamos de la belleza para que la vida vuelva a tener sentido... Pero ahora cómo agradecimiento.

(Bautista, 2016)

El presente texto da continuidad a las elaboraciones construidas en torno a la experiencia estética como instancia nuclear en la producción de subjetividades, de reproducción de la vida, y de construcción de realidad social. En particular pretende mostrar estrategias poco visibilizadas de agenciamientos que se despliegan en la cultura como formas de trabajo sobre la sensibilidad, entendamos este como arte, rito o producción cultural. Hemos venido construyendo una arquitectura conceptual que nos permita mostrar la dimensión tecnológica del quehacer artístico-cultural, no solo por la posibilidad de su acción sobre lo real, sino muy especialmente sobre el tipo de realidad a la que tiene acceso y con la cual trabaja. Es, pensamos, la falta de comprensión de su ámbito de trabajo, y no la naturaleza de sus dispositivos, lo que dificulta que pueda ser comprendido como trabajo tecnológico que opera sobre la realidad. Esto dificulta la plenitud de su desarrollo y señala las limitaciones de las teorías del arte que separan del hacer vital en el campo de lo simbólico como un idealismo abstracto.
Hemos encontrado de mayor utilidad una reflexión específica sobre las prácticas estéticas populares, con resonancias claves en la ritualidad y un desplazamiento hacia la perspectiva epistémica del hacer creador. En este sentido se avanza hacia una lectura de la agencia estética como forma de producción y trasformación de (es decir trabajo sobre) lo real, cuya cualidad principal seria la de operar como instancia de mutación entre los campos material y simbólico.

Se muestran en este sentido algunos dispositivos estéticos tradicionales y sus recreaciones populares para la mediación estética y la creación común de lo real que muestran este tipo de efectos que nos autorizan a pensar en unas tecnologías de lo sensible. Como cualquier otra tecnología sus efectos están signados por la intencionalidad de su uso, pero diremos por ahora que, en general, se orienta a la producción de una posibilidad existencial, es decir que es capaz de modelar la experiencia humana de vida movilizando sus condiciones simbólicas y materiales.

Ya Duvignaud (1970) y Durand (1988) ampliaron de forma muy importante el campo de debate referido al quehacer simbolizante y el lugar del imaginario, por ello llama la atención el modo como este saber queda, en el discurso epistémico y científico dominante como dato 
marginal, tan exótico como los pueblos estudiados. Sus teorías mostraron, desde una perspectiva crítica en la Europa de su tiempo, la dimensión científica (como parte de un régimen de verdad, entre otros), considerando entre otros los dispositivos rituales dramáticos en diferentes acervos culturales.

Esto significó, y así queda señalado en sus textos (Duvignaud, 1970), la puesta en cuestión del sujeto moderno como medida de la narrativa humana general, por lo que abre la posibilidad de agencias sobre lo real que responden a otras construcciones simbólicas con igual legitimidad teórica. Por supuesto, no omitimos el hecho de que tanto esos dispositivos, como los textos y archivos propios de esos acervos culturales otros, y especialmente sus portadores, quedaron igualmente postergados o diferidos frente a la episteme dominante. En muchos casos, pese al sincero interés de antropólogos y antropólogas por dar lugar y visibilidad a saberes de los pueblos estudiados, las estructuras semánticas contribuían contrariamente a su folklorización y a la suplantación de sus voces quedando oculto la mayor parte de ese saber. Es propósito de este texto abrir el debate en torno a la cualidad operativa, es decir tecnológica, de ciertas formas de saberes rituales, cuya comprensión puede informar una concepción muy potente de la experiencia estética contemporánea, su despliegue en el arte y la producción cultural, incidiendo en definitiva en la experiencia de vida colectiva.

\section{Tecnología - otra noción}

La constatación en el cuerpo material e inmaterial de afectaciones, transformaciones y provocaciones a partir de experiencias sensibles, así como el estudio de los efectos de las interferencias de las industrias culturales en los cuerpos subjetivos individuales y colectivos, hacen evidentes la posibilidad de interferencia intencionada de este campo. Un camino para estudiar las formas y las fuerzas que este tipo de dinámicas ponen en juego es la indagación sobre conocimientos específicos de experiencias populares de mediación cultural y estética. La música, la palabra, la imagen y el movimiento ritualizado (Imagen 2), como oficio o como pieza espectacular puede ejemplificar estas formas de generar huella y afectación, al ser utilizados de forma colectiva como en las fiestas populares, en manifestaciones espectaculares o incorporados en formas cotidianas de labor. Identificamos en estas prácticas elementos funcionales que sostengan su acción como tecnologías de lo sensible, como insumos para luego pensar su posible experimentación en este campo.

¿Qué es lo que hace la música en el cuerpo físico y en el cuerpo afectivo? ¿Cómo se enlazan las personas en el gran cuerpo danzante que es la procesión? ¿Que ocurre en la experiencia contemplativa de la imagen plástica o audiovisual? En cierto sentido este texto recupera una vieja pregunta en torno a la función poética para responderla desde la experiencia sensible, y en perspectiva popular-descolonial. Es un aporte más en la tarea de producción de un mundo intercultural en el que amplios acervos y potentes saberes que operan a través de la expresión estética como dispositivospara-la-vida tengan lugar.

Partiendo de una reflexión sobre la experiencia estética como conmoción productiva, en una operación de doble vía entre las dimensiones materiales e inmateriales (Perez Wilke 2017), el presente texto da cuenta de vías observadas y/o experimentadas de manipulación de estos procesos. Estos se muestran indisociables de algunas formas del saber que sostienen las agencias culturales comunitarias, es decir que la mediación estética constituye, justamente, un dispositivo de procesamiento que permite la metamorfosis productiva en la interface material-simbólica. Este proceso a su vez genera la constante territorialización y autopoiesis de estas relaciones, sus marcas y consecuencias, es decir un cierto territorio existencial. Vemos un proceso en el cual la mediación estética y/o cultural trabaja con y sobre la experiencia sensible dando lugar a la producción material-simbólica, describiendo el momento de la emergencia de la subjetividad a través de lo sensible.

Esta cualidad productiva y transformativa de lo real en la agencia estética es lo que permite comprenderla como un cierto tipo de tecnologías. En este sentido partimos de la propia cualidad productiva humana, de su capacidad y necesidad de crear-recrear el cuerpo y lugar de su existencia, no de los problemas de la estética, entendidos como la belleza o de la economía de las formas, ni siquiera de los problemas de lo sublime, sino solo cuando emerge de necesidades vitales. Se trata de observar el germen mismo de la creación en contextos populares para acceder a zonas oscurecidas de estos procesos y redescubrir ahí estrategias de creación de la realidad social.

En un texto de lo años 80, ya Enrique Dussel, para otros fines, había necesitado revisar la relación de las 


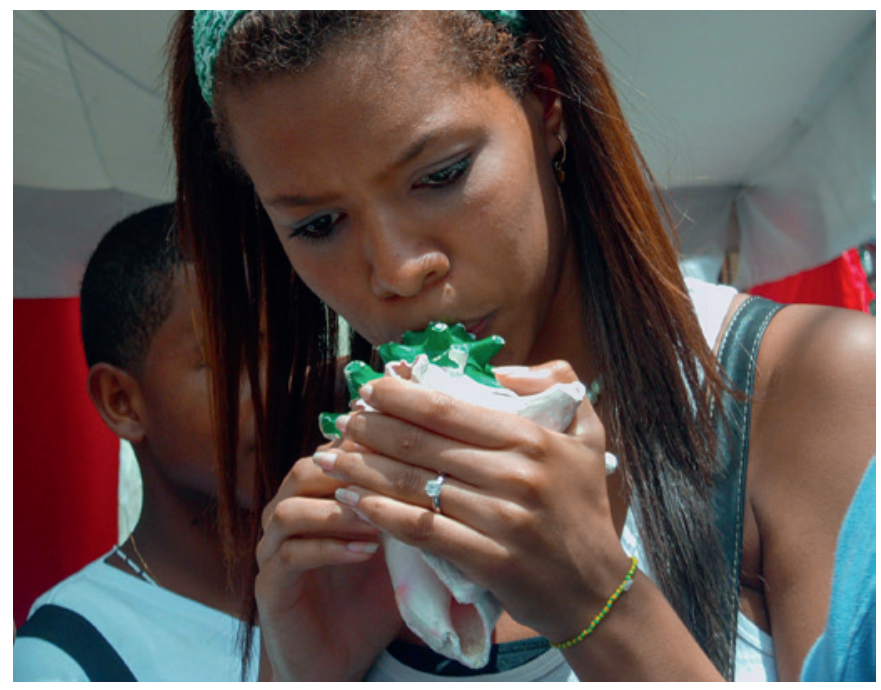

Imagen 2. Repique de San Juan. Junio de 2014. Parroquia La Vega, Caracas, Venezuela. Foto Inés Pérez-Wilke

nociones de tecnología, poiesis, estética, trabajo y producción, que esclarece nuestro punto de partida:

El desprecio por el trabajo manual, propio de esclavos, siervos, obreros, llevó a la filosofía a sólo ocuparse de la estética -la parte "más limpia" de lo que denominaremos poiética-. El trabajo manual era despreciado junto al trabajador. De lo que se trata es, justamente, de intentar comenzar a bosquejar por primera vez una filosofía completa del acto productivo, que reúna en sus fronteras actos aparentemente tan diversos como el lenguaje o la producción de signos, la tecnología con todas sus formas, las artes y los diversos tipos de diseño. (Dussel, 1984, p11)

Esta trama que pone en relación la actividad transformativa, tecnológica con su dimensión poética, muestra cómo solo a través de una escisión fue posible la existencia de una estética, al margen de la producción material, de la vida cotidiana, y del sostén de la vida. En el caso de las relaciones sociales que se dan en la medicina, en el teatro o en la escritura vemos formas específicas de afectación consciente, voluntaria sobre lo real-sensible a partir de un saber con estrategias específicas de cada ámbito, pero en-relación. En esta perspectiva, la consideración de formas de agencia plurales, no jerárquicas, les dan a todas las relaciones estéticas una dimensión política que va a tocar no solo las relaciones sociales, sino las relaciones con los contextos, y la concepción de formas de agencia no humana o no solo humanas. Dussel orienta desde temprano el primer paso de esta tarea:
Una historia crítica de la poiética o una destrucción de dicha historia es lo mismo. Se trata de demoler las interpretaciones vulgares, habituales, las tenidas por todos como evidentes. Es un ir refiriendo las partes al todo, los objetos al fundamento, lo abstracto a lo concreto; es un moverse dialécticamente de los entes a la totalidad, de la diferencia a la identidad, y aun superándola desde la exterioridad del oprimido. (Dussel, 1978 p.)

Dussel en ese momento se aparta de la discusión estética, tenida como abstracta y se orienta a una reflexión sobre el trabajo que construye la realidad social. De lo que se trata aquí es de revelar las profundas vinculaciones de la producción de experiencias estéticas en la fabricación de mundo, que más allá de la "conservación de las formas de existencia y a la presión que está ejerce contra la voluntad de potencia de la vida, constituye su impulso de producción de diferencia" (Rolnik 2019, p.176) como pulsión creadora de la vida. De modo que si, partiendo de aquel fundamento, nos aproximamos al estudio de las subjetividades emergentes, se confirma la necesidad de mejor comprensión de la dimensión estética de los agenciamientos sociales.

La fuerza de estas operaciones estéticas no condiciona su orientación ni su dimensión ética; pueden ser y son usadas también como tecnologías del control, de captura, de enajenación como ha sido explicado por Guattari y Deleuze (2010), en Foucault (2018) y en Machado da Silva (2006). En este trabajo se muestran tecnologías antiguas y nuevas, de la interface material-simbólica especialmente en su articulación ética, en tanto que potencia fértil a la vida de todos y todas, siguiendo el precepto descolonial y transmoderno (Dussel, 2017; Bautista, 2016; Rolnik, 2019). La definición del campo de actuación de estas tecnologías ya indica la perspectiva de acercamiento a la vida a la cual nos referimos.

Cuando hablamos de tecnologías estéticas de transformación de lo real, estamos refiriendo a una capacidad de mutación que implica una cierta sensibilidad; hablamos de un mundo y una realidad afectables por la acción poética. Se trata de una cualidad de la vida y de lo vivo, que es susceptible de mudanza y que por esta misma condición, acto y efecto alumbra lo nuevo. Llamamos a esta dimensión materia afectable (PérezWilke, 2019). Es la capacidad de esa materialidad de adoptar la forma que le imprime otra fuerza material o inmaterial, lo que permite a esa fuerza adoptar nuevas materialidades. Pero también la capacidad de ese 
cuerpo inmaterial de afectarse al contacto con fuerzas materiales percibidas. Alumbramos aquí ese momento y ese dispositivo, que en su sencillez encarna el momentum de la creación de realidad social.

Una tecnología de lo sensible sería entonces el conjunto de dispositivos y saberes que permiten movilizar lo intangible sobre una materialidad, y a su vez provocar la emergencia desde allí de nuevos intangibles simbólicos. Es un aparato plural que vemos operar a través de las formas rituales, culturales y artísticas, mediante los cuales, a partir de deseo, memorias, afectos, intuiciones se incide en la corporalidad del mundo, con la palabra, el sonido, el movimiento, la elaboración física, alcanzando la materia afectable. La producción de huellas, de movilización material retorna y emerge en resonancias y disonancias, de nuevos territorios y tiempos para pensar y en los cuales existir. La materia afectable es, entonces, una condición de la vida, que implica la sensibilidad ante la acción y las fuerzas que se generen a su alrededor o en su interior, con capacidad de interferencia y reversión. De este modo es un proceso que no solo transforma a través de lo que produce en lo(s) otro(s), sino también a quien lo produce, en formas de contagio y diseminación de lo que toca y es tocado.

Rolnik ha llamado, refiriéndose a esta dimensión de la vida, cuerpo vibrátil, instancia del saber del cuerpo que tendría una capacidad infinita de canalización del deseo para producción y transformación. En su transcurso, este materializa en la dimensión finita de la vida, de una manera singular y única: "Cuerpo sensible a los efectos de los encuentros de los cuerpos y sus reacciones: Atracción, repulsa, afectos, simulación en materias de expresión. (Rolnik, 2007, p 31). Esta idea, que acompaña toda su obra, presenta una instancia corporal, cognoscente y afectiva de procesamiento de las fuerzas que movilizan la realidad:

Activar el saber eco-etológico y expandirlo a lo largo de nuestra existencia: La experiencia del mundo en su condición de viviente, cuyas fuerzas producen efectos en nuestro cuerpo, el cual pertenece a esa misma condición y la comparte con todos los elementos que componen el cuerpo vivo de la biósfera. (Rolnik, 2019,175)

La materia afectable, como campo de trabajo de las tecnologías de lo sensible, esta de este modo expuesta a la dimensión estética de las operaciones rituales, de las acciones artísticas y de las estrategias culturales de producción de subjetividad en general. Así mismo a partir de ella se genera a través de la reversibilidad, un movimiento que toca los campos simbólicos a través de las huellas que la materialidad como las ondas sonoras de una música, de una palabra dicha o impresa, o de una performance, modificando ese campo difuso e inmaterial.

Este retrato brevísimo de antecedentes clave nos permite avanzar en un territorio más precario: la consideración del saber y el hacer popular en estos campos intangibles, tan asociados a la superstición, como faros que orientan la búsqueda de principios para una tecnología de lo sensible. Las acciones rituales de nuestros pueblos, con sus cantos y danzas, con sus pinturas rituales, en sus tejidos, encarnan el desarrollo de acciones que han movilizado los campos sensibles de la comunidad por generaciones. Mirados hoy, puesto que perviven, muestran principios tecnológicos olvidados o ignorados con capacidad para iluminar las prácticas creadoras en medio de las actuales tensiones de la producción simbólica. Más allá de la representación, y del mercado, encontramos en las tecnologías de lo sensible formas singulares de ejercicio estético-ético, agencias ecológicas, políticas y económicas para la vida en salud que se manifiestan a través de la producción estética.

\section{Brujería, rito y saber hacer:}

Las gungas ${ }^{1}$ ayudan al transporte hacia otro mundo donde el pasado, el presente y el futuro son parte de una misma procesión.

(Rodrigues, 1997)

Mirar desde esta perspectiva estética las formas en las cuales las fuerzas del campo popular conocen y movilizan esta dimensión de la vida, es una vía de estudio prácticamente desconocida de aproximación con una perspectiva poético-epistémica. Es decir que reinsertamos la experiencia sensible dentro del campo de la producción de conocimiento y a su vez legitimamos el hacer estético popular entre las operaciones vivas no solo de acción, sino también de formalización del mismo, con registros poco conocidos en la ciencia moderna, mostrando todo un campo de conocimiento por articular.

Evidencias de la vitalidad y fertilidad conceptual de estas prácticas se encuentran en numerosas acciones rituales y festivas del sur global. En ellas se identifican el

1 Gungas: Guardas de los congados brasileños y también las sonajas que los danzantes de la guarda usan en los tobillos. 
uso consciente y estudiado de movimientos corporales, danza, palabras más allá de su función comunicacional, estructuras sonoras y el rítmicas, el uso de indumentarias, la elaboración de objetos especiales como máscaras, bastones y figuras divinas entre muchos otros. Estos elementos están al servicio de un conjunto ritual intencionado, cuyas acciones tienen propósitos. La ejecución produce algo: invoca, expulsa, convoca, provoca, agita, calma, siembra, limpia; y esto solo es visible en ciertos registros. Es menester entonces, a fin de avanzar en la producción de un mundo realmente intercultural, hacer ejercicios de traducción y legibilidad.

Tal como explica Federicci (2010), mucho de aquello que desde la colonia y aún hoy dio en llamarse brujería, desde la misma época, fue reivindicada por movimientos como el Taqui Onqoi (Pérez Wilke, 2016), en Perú, mas tarde por escritores como en Aimeé Cesaire (1969), Retamar (2006) y por movimientos sociales en todo el continente. Estas prácticas son pensadas por los pueblos en relación a cuerpos de saber, y, sobre todo, de saber hacer sobre la realidad, como técnicas para relacionarse con otras fuerzas de la vida. Esos mismos saberes reclaman hoy otros lugares de visibilidad con su propio linaje epistémico y formas de registro (Imagen 3).

Oportuno ver esa cualidad de extrañamiento y poder que la palabra brujería connota en relación a la noción de tecnología que venimos articulando. Es decir que la brujería sería el conjunto de operaciones a partir de cierto saber (también propio) para la transformación de lo real de formas que les son desconocidas y abisales a occidente (ver también - Perez-Wilke, 2016).

Subrayamos aquí la dimensión fáctica del ejercicio cultural, y reflexionamos a partir de prácticas especificas y de narrativas locales sobre las mismas prácticas, pues es desde la operación hecha que podemos mostrar su trazo en un contexto occidentalizado. No se trata de poner de lado las creencias, sino de mostrar como la construcción de narrativas, la inversión performativa y las elaboraciones simbólicas asociadas a ellas, tienen consecuencias sobre lo real que merecen ser miradas. Si bien la potencia epistémica y productiva de este campo fue, en general, dejada de lado por la modernidad, con agravantes en las sociedades coloniales (donde además fueron perseguidas, prohibidas), muchas de las prácticas estéticas populares han sostenido las posibilidades generadoras saludables y espontáneas de los pueblos en general.

Estas formas de acceso y afectación de la realidad a través de lo sensible no han sido ajenas a Occidente, solo que este construyó una narrativa encubridora de sus propias creencias bajo una idea culta de razón y de arte. Recordemos aquí la crítica desarrollada en este sentido por Hinkelamert (2008) en torno al carácter religioso y fetichista de la modernidad y el capitalismo. No se trata de cuestionar la cualidad religiosa o la dimensión afectiva de cualquier forma de pensamiento en sí, sino justamente de hacerla evidente para poder asumir esa

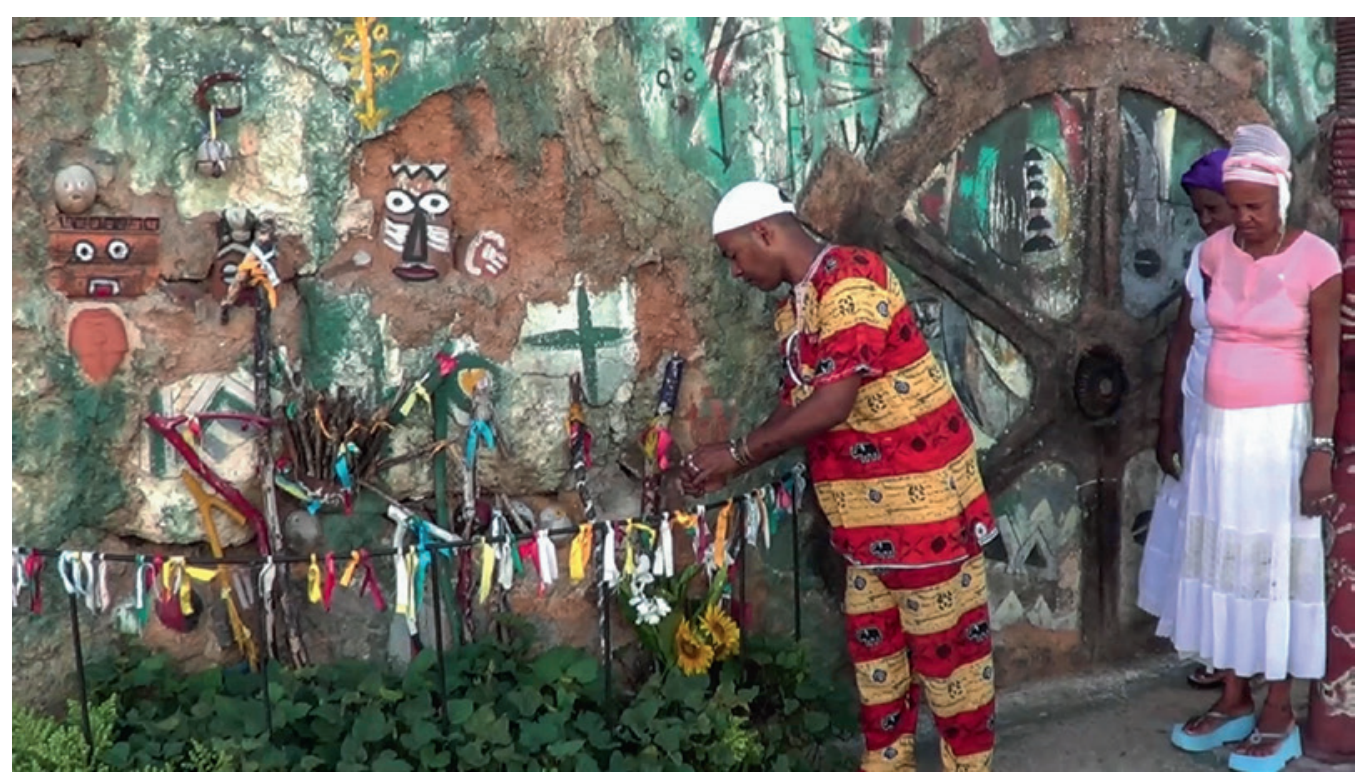

Imagen 3. Apertura de un Ñangareo. Matanzas, Cuba. Fotografía Francisco Pérez. Archivo de Investigación, 2014 
dimensión desde una reflexión situada, cuya pretensión de universalidad no solo sea dialogante, sino que contemple y se dirija al cuidado de la vida. Si podemos ver una clara relación entre estas agencias estéticas populares y lo que Occidente llamó artes, esta relación debe, tal como lo plantea Albán, identificar esta dimensión política de pluralidad: "El arte - actuando como mecanismo de auto-representación, de auto-significación y de construcción de nuevas simbologías - visibiliza, pone en evidencia la pluralidad de existencias que se encuentran y se desencuentran en el escenario multicolor de la contemporaneidad. (Albán, 2009, p. 91).

En esta perspectiva es posible avanzar en reconocer el alcance tecnológico de las formas operativas de los performances comunitarios como los espacios de danza colectiva, música, celebración, comidas, todas las formas de poesía oral, especialmente aquellas dedicadas a la curación, la fertilidad, la relación con la naturaleza, el equilibrio de género, la paz entre los pueblos. Reconocemos todas ellas como formas de elaboración, procesamiento y producción de lo real que operan en el hacer pasar contenidos materiales a simbólicos y viceversa. Como un cierto tipo de conocimiento poco documentado que a través de procedimientos performativos obtiene resultados moviendo campos de resonancias entre distintos planos de la realidad para las cuales las culturas han generado dispositivos de agencia, y de las cuales pensamos, la producción estética sería una muy importante.

El estudio y comprensión de formas rituales contemporáneas, populares han sido reconocidas en los estudios del performance como aportes para el estudio de las artes performativas actuales (Taylor y Fuentes, 2011) y las implicaciones de la performatividad cotidiana (Biao y Greiner, 1999). En esta perspectiva, pierde sentido la diferencia hecha entre las manifestaciones populares más estudiadas por la antropología y la etnografía rituales, y las acciones que la modernidad designa como artísticas, a no ser por los circuitos económicos que el arte ha producido, y por la mayor o menor distancia para con la vida cotidiana. En el plano de la elaboración simbólica y de las agencias estéticas la obra artística estaría trabajando en este pivote materia-simbólico, siempre que responda a contextos reales y situados. Así se compromete con la movilización del entorno como espacio operativo de la sensibilidad contemporánea, con potencialidades epistémicas que la noción de tecnologías de lo sensible consigue. Esto puede constituir una transformación profunda, ya que está comprometida la propia experiencia espacial y corporal de agencias alteritarias, dialogantes que la re significación de la palabra brujería interpela y que en definitiva es lo que podría permitir aperturas hacia nuevas formas de relación intercultural de vida.

\section{Operaciones estéticas - Tecnologías del flujo material-simbólico}

En una lectura de Rolnik vemos que la operatividad de las prácticas artísticas en la instancia que ella llama estético-clínica puede ser recuperada para lograr desplazamientos y autonomías populares en el despliegue de la pulsión creadora y transformadora:

La reapropiación de la pulsión depende de reapropiarse igualmente del lenguaje (verbal, visual, gestual, existencial, etc.) lo que implica habitar el lenguaje en los dos planos que lo componen: el de la expresión del sujeto y el de fuera del sujeto que le da movimiento y transforma el lenguaje. Esto depende de lanzarse en un proceso de experimentación, movido por la tensión de la paradoja entre ambos - lo que es indispensable para que la pulsión pueda guiar el deseo hacia las conexiones que le permitan crear algo en el que los afectos encuentren su expresión. (Rolnik, 2019, p 120)

Queda en evidencia que esta apropiación de los lenguajes implica, además del estudio de los archivos y tecnologías estéticas populares, retomar fuentes, medios, y efectos que en algún momento fueron subsumidos en lo que hoy llamamos hoy llamamos arte. Este campo y más ampliamente de la producción estética son campos heterogéneos que ciertamente han convocado a la exploración de territorios creativos de cierta fragilidad. Sus iteraciones y elaboraciones materializan, y a la vez provocan, búsquedas y experimentaciones de/en las mas íntimas zonas de las pulsaciones humanas mostrando de manera más clara la naturaleza sutil de la tecnología a la que nos estamos refiriendo. Transita desde territorios muy personales, intuitivos, pre-verbales a nodos colectivos de sensibilidad y narrativas, a menudo en disputa. En este sentido comporta una relación cultivada con la operación material-simbólica en cualquiera de las áreas, lo cual implica responsabilidad para y por el desarrollo de lo ritual contemporáneo.

Todo lo que fue amarrado en las categorías de arte, artesanía, arte popular, toda obra que opera sobre la sensibilidad: el canto, la pintura, la arquitectura, la escultura, las artes de la escena, así como las muchas formas de fabricación: cestería, alfarería, tejido, 


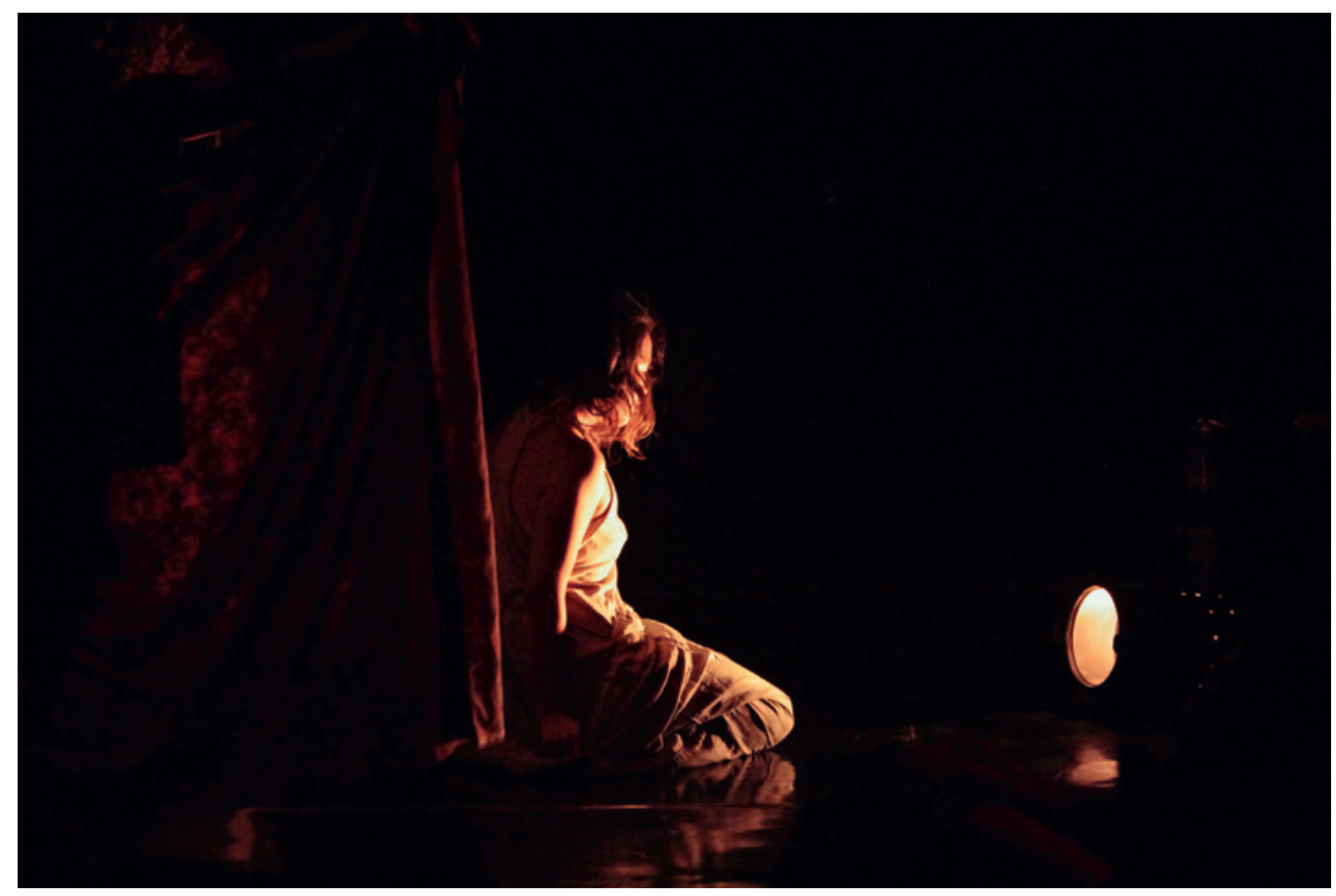

Imagen 4. Tríadas Improvisación al Borde. Grupos Semeruco Impro. 2019. Foto Isadora Yanes, Archivo del Grupo.

costura; pueden considerarse formas de hacer panal, de construir, crear, sostener un contexto a ser ocupado, de hacernos nido, de desplegar condiciones para la vida humana ante los ojos. En su potencia esta misma producción sería la forma de habitarla: la paradoja de una vida que se despliega para vivirla, de un espacio que se construye para recorrerlo; paradoja tecnológica-sensible de la fabricación incansable de mundo.

\section{Artefactos para una tecnología de lo sensible}

El arte re-configura y problematiza las espacialidades.

(Albán, 2008, 108)

Viendo en detalle expresiones de la lógica fabricante y transformativa de los pueblos, que articula entre los campos materiales y simbólicos, encontramos que esta identificación que se hizo entre arte popular y brujería esta vinculada a la forma que se organiza como saber para producir resultados no previsibles por una lógica causal, porque las tecnologías sensibles operan de otros modos, por resonancia, por diseminación, por analogía, por iteración orgánica, que es preciso conocer y comprender mejor.

La tarea que venimos desarrollando es el registro, la experimentación y desarrollo de estrategias de estas tecnologías de la sensibilidad, principalmente en el campo de las artes escénicas, a propósito de nuestra práctica en improvisación colectiva e interdisciplinar. En este caso vamos a mirar la producción en una clave más amplia con base en principios que tocan diversas formas de operación en agenciamientos colectivos y registrar movimientos e implicaciones de restituir el campo estético como campo de saber y de producción:

Estructura ritual: Nos referimos a un conjunto de elementos que respetados permiten generar un espacio-tiempo interior y común, marcar un contexto con cualidades singulares. Lo ritual genera una tensión entre la rigidez de estructuras que se repiten, que deben repetirse y formas flexibles, que permitan al mismo tiempo sostener y hacer pasar lo vivo, lo nuevo, como creación social (Imagen 4). No se trata de establecer una sinonimia entre arte y ritualidad, se apunta a identificar elementos tecnológicos de manipulación de lo real comunes y zonas superpuestas, a través de los cual se hacer mutar elementos de la realidad y a nosotros mismos.

Esta acción humana performativa tiene la capacidad de sacralizar determinados tiempos y espacios, generando posibilidad de que las interacciones materiales que allí ocurren estén ya en un plano simbólico. A través de marcas en el espacio y el tiempo, de la cualificación sonora y/o gestual, se altera y/o se sostienen narrativas de la comunidad en lo real. La ritualidad, aunque puede no tener espectadores, da lugar a la construcción de 
un espacio escénico y sus cualidades, es decir, protege lugares privilegiados de visibilidad, que condensa miradas y expectación, a raíz de lo cual se transforma en un espacio de poder, de atención y cuidado no solo estético, sino también ético. Por ello, en la mayoría de los pueblos, estos procesos rituales implican períodos de formación, conocer en detalle técnicas adecuadas que cuidan la celebración o el evento, que puede contener elementos complejos. La actuación en este campo conlleva acciones con consecuencias y vinculaciones en el entorno, comporta riesgos en su uso displicente, puesto que mueve imaginarios, y sensibilidades con frecuencia masivos.

Intervención a través del sonido: Una de las tecnologías estéticas más ampliamente usadas y reconocidas, es la sonora. Sus vibraciones físicas atravesando casi todo tipo de materias, y el universo imaginal que moviliza, hacen del sonido, y de sus conjuntos articulados, sean reconocidos como música o no, poderosos artificios. Actualmente su uso en la publicidad, en los noticieros, en ceremonias religiosas de diverso tipo y otras estrategias de las industrias culturales delatan su instrumentación como tecnología de conmoción sensible desde una perspectiva psicologicista y conductual.

Queremos referirnos aquí a la posibilidad de contra-interferencia que ofrece ella misma como instancia de comunicación y vivencia comunitaria, en tanto que puede ser usada para propiciar y evidenciar los límites de la experiencia subjetiva individual y evidenciar los tránsitos de subjetividades colectivas, inclusive mas allá de lo humano. El despertar de la consistencia acuosa del cuerpo, por la música, y su analogía al comportamiento de la electricidad, más allá de su transformación literal en impulsos eléctricos que recorren el tejido vivo.

Más allá de la no tan conocida existencia del tratado romano de Boecio, que es un antecedente europeo prácticamente olvidado, este saber habita en las manifestaciones populares alrededor del mundo. Músicas de sanación, de parto, de caminata, de trabajo, de comunión humana, de comunión con lo sagrado, testifican un saber no traducido. Vemos ejemplos de cantos curativos entre los akawaio, los pumé, y kariñas por mencionar algunos pueblos de la Orinoquía. Se trata de articulaciones precisas, de entonaciones, ritmos y palabras hechas para comunicarse en distintos planos a diferentes interlocutores, a la enfermedad, a los antepasados, al interior de la persona enferma, que dialogan con las fuerzas puestas en juego.
Entre los pueblos afrodescendientes, vemos el uso comunicativo de la percusión y el canto. Este en primera instancia sincroniza a la comunidad y la enlaza, pero luego, en estas tradiciones es la música a través de la cual desciende lo sagrado. Se convierte literalmente en un canal de la comunicación entre los mundos de Orun y el Aye. Esta conmoción que la vibración sonora provoca en la materia afectable, es una experiencia más allá de sí, como el propio canto, una emanación, al tiempo que constituye una atmosfera por la cual se es atravesado y que configura la hermandad con el todo. Hasta tiempos relativamente recientes esa función y esa hermandad seguían habitando la música, como conversamos con Juan José Bautista:

Pónganse ustedes a analizar el tipo de letras que existían en las canciones de antes. No estoy diciendo de hace 300 ó 400 años, estoy diciendo digamos de hace 50, 60, 70 años. En las zambas argentinas, por ejemplo: Está triste y su tristeza ¿A quién se la canta? A la luna: "Ay lunita tucumana" canta a la luna, al río, al agua, al lago... A los árboles: "A los bosques yo me interno a echar mis penas llorando" "y los bosques me contestan..." y ahí va, y le cuenta... Y los bosques le responden, le apapachan, le calman, y sale bien después de eso. (Bautista, 2016, p. 8)

Incorporación intensa y extensa: Los cuerpos vivos encarnan una materialidad transformativa privilegiada, aunque ella se extienda también al mundo mineral y a las fuerzas físicas. El trabajo en esta corporalidad presente y viva de lo teatral da forma a la experiencia incorporada significante, y desarrolla una trama performativa que constituye por sí misma una agencia sobre lo real. Pueden identificarse en las más diversas culturas acervos y archivos de saberes del cuerpo, los cuales, si son tratados como tecnologías activas y no como registros del pasado, mantienen toda su vigencia y su cualidad operativa. Cómo artesanía de movilización individual y colectiva, implica esquemas corporales diferenciados, danzas, ejercicios psico-corporales como el yoga, artes acrobáticas, juegos y pruebas físicas hasta las configuraciones de una corporalidad cotidiana singular. Los cuerpos en movimiento constituyen una fuente de emanación de imágenes, de apariciones y sentidos: aquello que alargan las extremidades más allá de sí; las sutiles variaciones en el movimiento de la columna; el manejo intencionado del peso de un punto a otro del cuerpo (Imagen 5). 


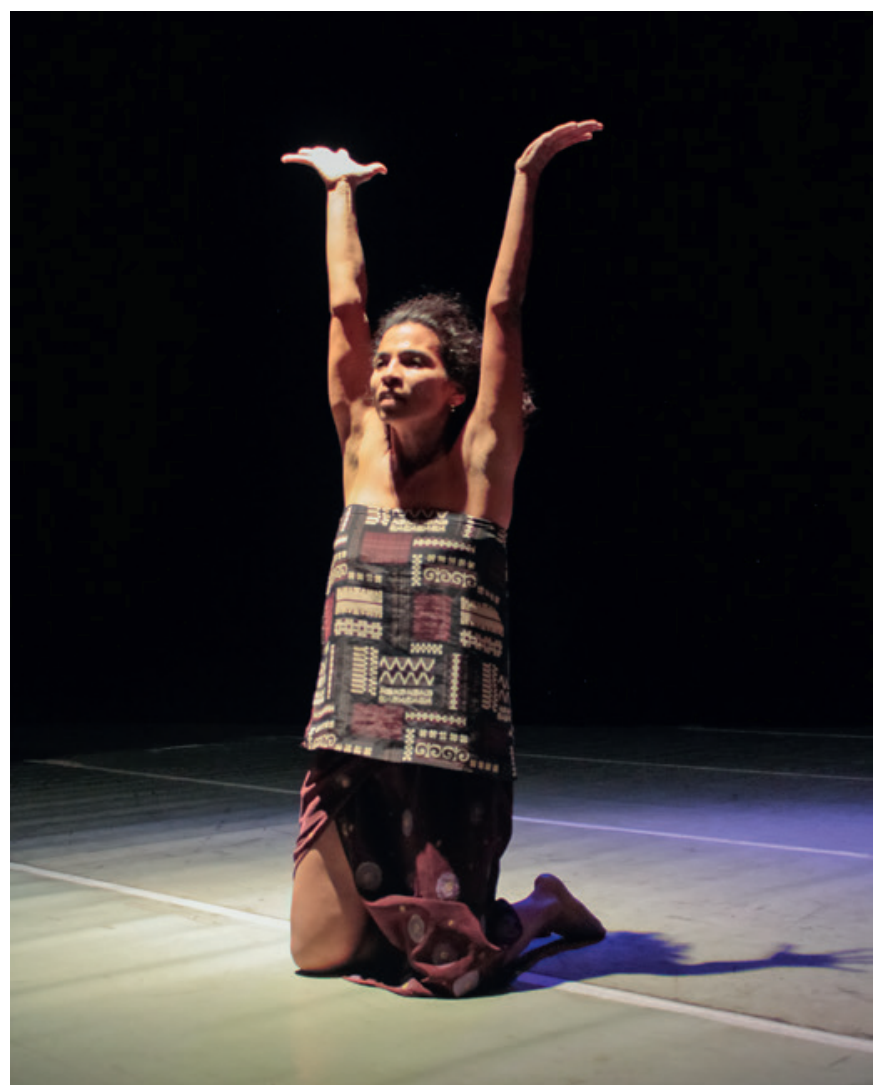

Imagen 5. Semeruco.GIT en Tríadas: Improvisación al Borde. 2020

Si las energías de la tierra y las del cielo se encuentran en el ritual, estas se materializan en el cuerpo, eje de esa alquimia. Dice Graziela Rodrigues: “El cuerpo entero es el fruto del enlazamiento del cotidiano con la festividad" (1997, p.125), y propone una escuela del movimiento ritual, segura de los saberes que allí se esconden. Con Chillemi (2015, p.62) vemos un ejemplo de este tipo de experiencia: "Realizar el movimiento en 'diferentes tiempos', modifica la percepción del espacio recorrido, pudiendo llegar a la vivencia de recorrer un espacio 'infinito', con apenas el desplazamiento de una parte del cuerpo. Espacio personal, parcial, total, compartido". Esta vivencia de la temporalidad, permite la dilatación o contracción del tiempo, de circularidades, de manipulación de ese factor vital a través del ritual y del movimiento.

La fuerza de los elementos estéticos y performativos del movimiento, la gestualidad y la agencia corporal de relación con el mundo, actúan en los espacios públicos como formas políticas. Puede verse en la urbe, en los vectores de los desplazamientos de jóvenes patinadores, o ciclistas, o en la gestualidad que resuena con determinada geografía, o en nuevas formas de ritualidad de género, o festivas, cuya intencionalidad puede ser cultivada.

Conjunción de lo heterogéneo: Nos referimos aquí a las estrategias de acumulación de fuerzas a partir de la sumatoria de flujos energéticos o corrientes, como las procesiones, comparsas de danza y corales, por poner algunos ejemplos, con su elemento clave: lo masivo. Pero también lo encontramos en la fusión de fuerzas expresivas, de formas cada vez mas complejas como: polirritmias y polifonías, danza-teatro, hip hop son todas formas de hacer confluir organizaciones ya conocidas en agenciamientos mayores, flujos creativos mas allá de lo personal en subjetividades colectivas que aparecen como nuevos textos.

La experiencia estética ocurre del encuentro de lo heterogéneo (Pérez-Wilke, 2017), se alimenta de la fuerza colectiva, colocando la voluntad de agencia en un espacio intermedio, inclusive si se trata solo de aquel entre el escritor y el lector, con mayor potencia en las acciones colectivas. Estas, a la vez, conducen y permiten la permeabilidad de nuevas corrientes. Esto pasa por una cierta conciencia colectiva de participantes, músicos, danzantes y no danzantes, no necesariamente racional, para su disponibilidad más allá de sí. De un cuerpo al cuerpo masivo, la unificación del tempo vital, de la respiración colectiva, es una cadencia común a la que llegar, hacer mutar y de la cual también salir.

En el caso de las fusiones, vemos allí formas de los agenciamientos a los se refieren Guattari y Deleuze (2010). Aquellas articulaciones inesperadas de lo heterogéneo que realizan la existencia de algo nuevo, de algo distinto a la suma de sus elementos, y lo hace presente. En este sentido podemos pensar en la conjunción de distintos tipos de agentes, como individuales y colectivos, o humanos y no humanos, pero también en la conjunción de diferentes tramas de sentido como en el caso de las búsquedas estéticas propuesta por los movimientos de la disidencia de género.

En general la conjunción como tecnología obedece al principio heterológico, que muestra cómo en el sustento de la vida opera procesando y a la vez produciendo la diferencia, en ejercicio permanente de conjunción, fusión y desplazamiento creador.

Modelado de la materia plástica: La arquitectura tradicional, la alfarería, la talla, la muñequería, la estatuaria, son formas inmediatas de modelación de la materia-vacío como tecnología transformativa. Pero también 
la música modela el sonido, y también el cuerpo es trasformado plásticamente por la danza. Son procesos que hacen pasar un imaginario emergente, de necesidad o deseo en estado naciente, hacia materialidades compartidas en el espacio intersubjetivo con algun tipo de presencia.

La fisonomía cultural de los pueblos se fabrica en estas instancias de forma orgánica; en el sentido que responde a sus patrones de diseño, y también a un acumulado de acciones y prácticas que en su conjunto modelan un mundo habitable para aquellos que lo han producido. Pensamos en todos los paisajes transitados y habitados que hacen visibles sus trazas modeladas, surcos de siembra, casas, plazas, colores que muestran los desarrollos artesanales derivados de su vida cotidiana, y espiritual.

Las formas de modelaje como vestuario y las del trabajo performativo que modelan los cuerpos alimentan las posibilidades transformativas del ser, hacia la/el otro/a. Rolnik refiere la creación de mundo virtuales (2019), pero en ella lo virtual se hace presencia, finito ilimitado. Hablamos también de la plasticidad material del espacio escénico, que se extiende a acciones que aparecen desde la plaza o la calle hasta el teatro o el espacio virtual que brinda una cámara. “El espacio de los públicos crea memorias y sentidos de existencia, posibilita la re-invención permanente de los mismos, y hace que la vida fluya en una suerte de dinámica donde las retinas se afectan, afectando a su vez los recuerdos" (Albán, 2008, p. 109). En las diversas formas de producción de hábitat, se revela la plasticidad del mundo.

\section{Prospección}

La formalización de experiencias tangibles en el campo de las prácticas estéticas indica que no hay producción que sea solo material o solo simbólica, que se operan constantes metamorfosis no visibles, o encubiertas, de producción de realidad, al alcance de todos y todas por medio de determinados tipos de conocimiento. De esta manera encontramos que lo que realizan estas tecnologías estéticas o de lo sensible se refiere a la producción de una posibilidad. En el sentido de que, entre las muchas contingencias, las fuerzas creadoras logren cada vez una configuración de existencias concretas, expresadas en la experiencia. Por ello es una necesidad clave sostener permanentemente la vinculación ético-estética, pues le reconocemos el poder destructivo y el poder creador como fuerzas en tensión permanente; la vida sobre la tierra es una posibilidad y no un destino. Vemos en las herramientas poéticas una vía de protección y cuidado de la vida y en este sentido, tanto el desarrollo tecnológico-poético, como una amplia divulgación de saberes y tecnologías populares ético-estéticas parecen indispensables.

En este sentido asumimos la responsabilidad de aportar una mirada prospectiva diciendo que, a partir de la idea de tecnología sensible, con apoyo en el estudio del saber ritual y en la experimentación estética, es posible contribuir con la democratización de la actividad creadora de mundos a partir de su dimensión sensible. Entre las rutas de desdoblamiento de estos temas, vemos el reconocimiento de la dimensión ritual en clave contemporánea y no dogmática; proponemos profundizar el estudio sobre el sonido y la corporalidad en relación con la experimentación del espacio-tiempo; así como con la plasticidad de lo real como forma de saber y de potencia tecnológica para la vida.

\section{Referencias}

Albán, A. 2008. "Arte y espacio público: ¿un encuentro posible?", en Revista CALLE 14, número 2.

. 2009. "Artistas Indígenas y

Afrocolombianos. Entre las memorias y las cosmovisiones. Estéticas de la Re-existencia", en Arte y estética en la encrucijada descolonial / compilado por Zulma Palermo - 1aㅡ ed.- Buenos Aires : Del Signo.

Bautista, JJ. 2014. ¿Qué significa pensar desde América Latina? Madrid: Editorial Akal.

2016. Condiciones para pensar una estética transmoderna en perspectiva decolonial. Entrevista con el grupo Caracara. Escuela de Pensamiento Descolonial. Caracas, Venezuela.

Boal, A. 1996. El arcoíris do desejo. Rio de Janeiro: Civilização Brasileira.

Biao, A.y Christine Greiner (Org.) 1999. Etnocenologia: textos selecionados, organizadores. - São Paulo:

Annablume,

Cesaire, A. 1969. Une Tempête. Paris: Seuil.

Chillemi, A. 2015. Movimiento Poético del Encuentro. Buenos Aires: Ediciones Artes Escénicas. 
Durand, G. 1988. La imaginación Simbólica. Buenos Aires: Amorrortu editores.

Dussel, E. (1984). Filosofía de la producción. Bogotá, Colombia: Nueva América.

Duvignaud, J. 1970. Spectacle et Société. Paris: Editions Denöel.

Federicci, S. 2010. Calibán y la Bruja. Madrid:

Traficantes de sueños.

Fernández Retamar, R. 2006. Todo Calibán. Alba. La Habana: Fondo Cultural.

Foucault, M. 2018. La sociedad punitiva. Madrid: Editorial Akal.

Hinkelamert, F. 2008. Hacia una crítica de la razón mítica. Caracas: El perro y la Rana.

Lezama Lima, J. El reino de la Imagen. Caracas: Biblioteca Ayacucho.

Perez-Wilke, I. 2019. "La experiencia estética popular: elementos para la acción descolonial", en Revista Communiars. Nro.2. 2019: 98-115
2017. La mujer Pueblo y sus relatos de vida.

Caracas: Ediciones UBV.

(2016) “La experiencia de I@s Otr@s. Claves para una heterología Suramericana". Tesis doctoral Aprobada en la Universidad Bolivariana de Venezuela 2016. S/Publicar.

Rolnik, S. 2007. Cartografía Sentimental. Porto Alegre: Sulina, Editora de UFRGS. 2019. Esferas de la Insurrección. Buenos Aires: Tinta limón.

Rodrigues, G. (1997). Bailarino - Pesquisador interprete: processo de formação. Rio de Janeiro: Funarte.

Silva, J. 2006. As tecnologías do imaginario. Porto Alegre: Sulina.

Tylor, D. y Marcela Fuentes. (edits.) Estudios avanzados de performance. México : FCE, Instituto Hemisférico de Performance y Política, Tisch School of the Arts, New York University, 2011 